\title{
Towards safe and optimized separation processes, a challenge for nuclear scientists
}

On April 22-24, 2015, the first International Workshop of the FP7 European Collaborative Project SACSESS (Safety of ACtinide SEparation proceSSes) took place in Warsaw, Poland. The conference entitled "Towards safe and optimized separation processes, a challenge for nuclear scientists" was co-organized by the SACSESS Coordination Committee led by the Project Coordinator, Dr Stéphane Bourg (CEA, France), and by the team from the Institute of Nuclear Chemistry and Technology (IChTJ, Warsaw), one of the partners of the SACSESS project, led by Prof. Jerzy Narbutt. The scientific sessions were held at the Primate Palace on Senatorska street.

With nearly 120 attendees from 15 countries, representing 45 research organizations, the Workshop was one of the biggest international conferences dedicated to actinide separation processes. It was the occasion to present widely the activities carried out in the Project by the EU research teams, but also to position them in a wider international context thanks to the participation of researchers from USA, Russia, Japan and the Republic of Korea. 40 oral talks and 26 posters covered all the topics linked to the development of hydrometallurgical and pyrometallurgical separation processes for actinide recycling, such as basic research, process modeling, radiolytic stability of solvent extraction systems, transfer kinetics, material studies, analytical developments, lab-scale tests, spiked tests and hot-test demonstrations, etc.

The Workshop was the corner stone of our programme for the dissemination and education. Indeed, in addition to the high visibility given to the work performed in SACSESS, the floor was widely given to the young generation, both for the oral talks and the chairmanship of the sessions. Therefore, our young researchers appeared closely in interaction with many recognized international experts with whom they could exchange ideas during the entire conference.

The Workshop presentations are available on the SACSESS website (http://www.sacsess.eu), together with the pictures of the first day. The present issue of "Nukleonika" consists of seventeen original papers based on certain Workshop presentations, preceded by a general introductory paper devoted to the aims and the research activities of the SACSESS project. We hope that the readers find it an interesting and useful read.

Stéphane Bourg and Jerzy Narbutt Guest Editors 